Mathematical and Computational Applications, Vol. 16, No. 3, pp. 659-668, 2011.

(C) Association for Scientific Research

\title{
INFRARED FREE ELECTRON LASER, RESONATOR PARAMETERS OPTIMIZATION WITH GENESIS, OPC AND GLAD CODES
}

\author{
Hatice DURAN YILDIZ \\ Dumlupınar University, Faculty of Arts and Sciences, Physics Department, \\ Merkez Kampüs, 43100, Kütahya / Turkey \\ hyildiz@dumlupinar.edu.tr,hyildiz@cern.ch
}

\begin{abstract}
A wide range-infrared free electron laser (IR-FEL) is being constructed for science and industrial applications in the Accelerator Technologies Institute, Ankara University. In the facility, in order to obtain electron beam with energy $40 \mathrm{MeV}, 4.5$ cell RF cavities are considered. IR-FEL system covers $2.5-250 \mu \mathrm{m}$ wavelength range that based on 15-40 MeV e-linac and two undulators to generate up to $250 \mu \mathrm{m}$ coherent infrared radiations. In this study, IR-FEL Resonator System parameters, mirror parameters, undulator parameters besides mirror diffraction losses are calculated and optimized with analytically and by using Optical Propagation Code and GENESIS 1.3. These Codes are also used to simulate beam behavior inside the resonator and along the undulator while GLAD Code is used for mirror diffraction losses.
\end{abstract}

Keywords: Infrared Free electron laser, Optical cavity, Mirror diffraction loss

\section{INTRODUCTION}

A wide range IR-FEL is being constructed for science in the Accelerator Technologies Institute, Ankara University within the collaboration of Dumlupınar, Niğde, Süleyman Demirel, Uludağ Universities for the IR-FEL Project Technical Design framework. The IR-FEL System consists of an electron linac, 15 - $40 \mathrm{MeV}$ energy range, with two optical resonators in order to obtain 2.5 - 250 micron infrared free electron laser. Undulators inside the resonator system will have periods of $2.5 \mathrm{~cm}$ and $9 \mathrm{~cm}$.

The facility is being located in the Ankara University, Gölbaşı Campus and the commissioning is planned to be by the middle of 2013. The Accelerator Facility is also includes the Bremstrahlung System with the same source of thermionic gun and initial energy - $250 \mathrm{KeV}$.

Electron beam with tunable energy in $15-40 \mathrm{MeV}$ can be injected to undulator-1 and undulator-2 lines independently. In order to obtain high quality FEL, electron beam should have high peak current [1], thus for effective acceleration, the beam needs to be squeezed and raised the velocity [2]. Beam has about 500 ps length after gun needs to be compressed using two step buncher called subharmonic and fundamental buncher cavities that operates 260 and $1.3 \mathrm{GHz}$, respectively. The bunch after injector is compressed up to $10 \mathrm{ps}$ and has $250 \mathrm{keV}$ energy. A booster linac will be used to accelerate beam up to $1-2 \mathrm{MeV}$. As an average beam current, $1.6 \mathrm{~mA}$ is taken into account. 


\section{MATERIALS AND METHODS OF OPTIC RESONATOR PARAMETER CALCULATIONS}

An optical resonator is an arrangement of optical components which include different kinds of mirrors at both ends of resonator system and undulator which is placed at the center of resonator. Optical components allow beam of light to circulate inside the closed path-resonator system. Two optic resonators are selected in the IR-FEL System with same length that houses planar two undulators have different periods. Symmetric and nearly concentric resonators are chosen in FEL System. For this selection, stability parameters played crucial role, they are obtained and placed in the stability figures.

\subsection{Undulators, Magnet Selection and Parameters}

Scanning large wavelength region is possible with wide tunable undulator's strengths as well as wider tunable electron beam energy according to FEL wavelength relation. Therefore, $25 \mathrm{~mm}$ and $90 \mathrm{~mm}$ undulator periods have been chosen for scanning 2.5 250 microns FEL wavelength with $15-40 \mathrm{MeV}$ range electron beam in the IR-FEL System. For 2 - 250 micron wavelength laser light, considered two planar undulator magnets satisfies the $0.2-0.8$ and $0.7-2.5$ intensities.

The schematic view of the undulator is shown in Figure 1. Since very high magnetic properties is presented by $\mathrm{SmCo}$, SmCo magnet material hybrid-type undulators with iron poles are chosen. SmCo is more brittle and thermoduric than the other undulator materials. Fundamental parameters of undulators are given in Table 1.

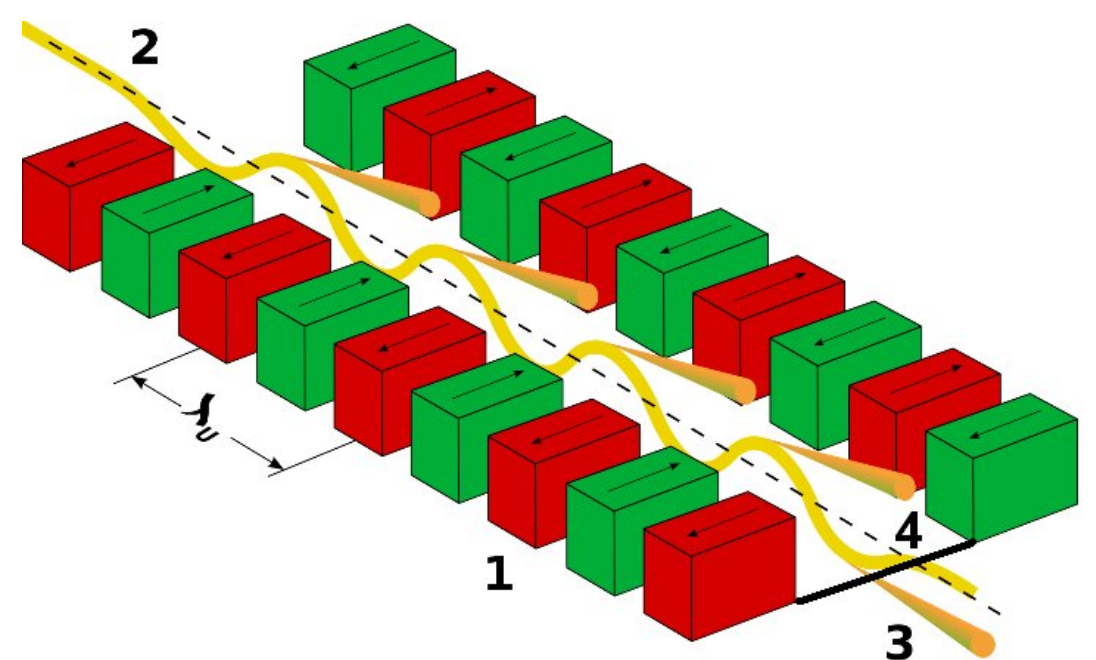

Figure 1: Schematic view of the undulator-1 where 1 shows undulator magnets, 2 shows electron beam, while 3 shows laser radiation and 4 shows undulator gap

The numbers of the undulator poles were determined with optimum gain and optimum intra cavity power. Current studies show that the strength for undulator- 2 is limited at 2.5 micron but in the future, it can be increased up to 3 . The strength of undulator- 1 is limited by the minimum gap. The beam pipe is considered to have $1.5 \mathrm{~cm}$ radius between the undulator-1 layers. 
Table 1: Fundamental parameters of SmCo type undulators

\begin{tabular}{|l|c|c|}
\hline Parameter & Undulator-1 & Undulator-2 \\
\hline Magnet Material of the undulator & SmCo & SmCo \\
\hline Period Length $(\mathrm{mm})$ & 25 & 90 \\
\hline Number of periods & 60 & 40 \\
\hline Magnet block dimensions(width*height*thickness)(mm) & $74 * 26 * 10.5$ & $90 * 90 * 35$ \\
\hline Steel pole dimensions (width*height*thickness $)(\mathrm{mm})$ & $74 * 18 * 2$ & $70 * 20 * 10$ \\
\hline Magnetic gap $(\mathrm{mm})$ & 15 & 40 \\
\hline Effective field $(\mathrm{T})$ & 0.3591 & 0.4205 \\
\hline $\mathrm{K}_{\mathrm{rms}}$ & 0.71 & 2.5 \\
\hline
\end{tabular}

\subsection{Resonator and Mirror Parameters with Analytical Calculations and Codes Comparison}

The distance of mirrors is related with the electron bunch repetition. In IR-FEL System, the bunch repetition rate is obtained $77 \mathrm{~ns}$ while corresponding mirrors distance is found $L_{c}=11.53 \mathrm{~m}$ by using the equation, $\mathrm{L}_{\mathrm{c}}=(\mathrm{cT}) / 2=\mathrm{c} /(2 \mathrm{f})$ where $\mathrm{c}$ is the speed of light and $\mathrm{f}$ is frequency of the bunch, $\mathrm{f}=1 / \mathrm{T}=13 \mathrm{MHz}$. Oscillator IR-FEL System consists of 2 mirrors at both end of the resonator. These are mainly spherical mirrors with one has small hole at its center to get laser out of the system. Symmetric and concentric resonator types are chosen since they have several advantages such as small waist, big mirror spot. Schematic view of IR-FEL Resonator System is displayed in Figure 2.

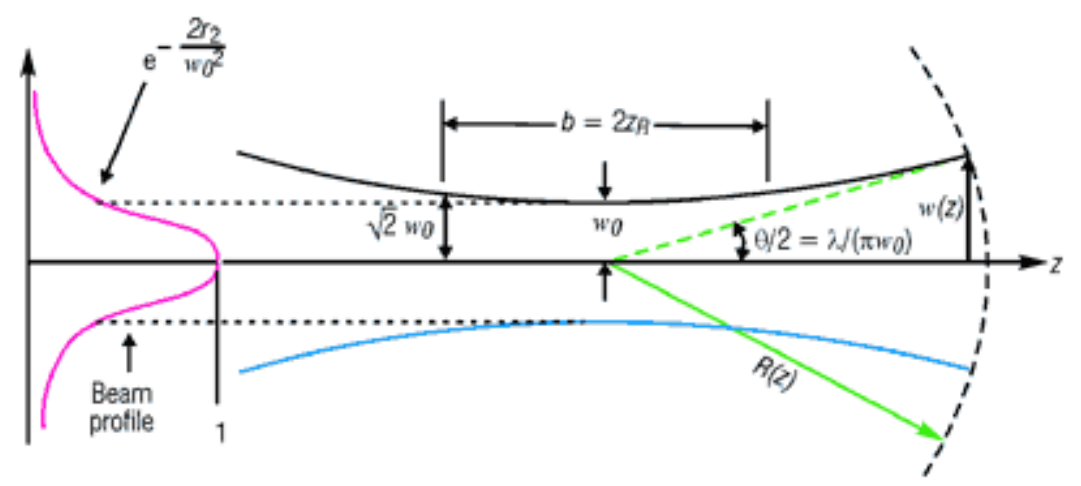

Figure 2: Schematic view of the IR-FEL resonator medium

Resonator parameters in our IR-FEL System are studied with the Genesis 1.3 and Optical Propagation Codes (OPC) [7-8]. Genesis simulates undulator part in the resonator while OPC simulates the beam behavior from the end of the undulator through mirrors by using Modified Fresnel Integral propagation. From the simulations, we have obtained TEM Modes that suits in IR-FEL Resonator. By using the equation $\mathrm{W}_{\mathrm{pl}}=\mathrm{C}_{\mathrm{pl}} \omega_{0}$, where $\mathrm{C}_{00}=1, \mathrm{C}_{01}=1.5, \mathrm{C}_{10}=1.9, \mathrm{C}_{11}=2.15, \mathrm{C}_{20}=2.42, \mathrm{C}_{21}=2.63$, calculating beam waist, 


$$
\omega(z)=\omega_{0}\left[1+\left(\frac{\lambda Z_{R}}{\pi \omega_{0}^{2}}\right)^{2}\right]^{1 / 2}
$$

One can obtain $\omega_{0}=0.002891 \mathrm{~m}$ and Rayleigh length, $\mathrm{Z}_{\mathrm{R}}=0.97$ for undulator- 1 by using,

$$
Z_{R}=\frac{\pi \omega_{0}^{2}}{\lambda_{R}}
$$

and, or by using stability parameters,

$$
Z_{R}^{2}=\frac{g_{1} g_{2}\left(1-g_{1} g_{2}\right)}{\left(g_{1}+g_{2}-2 g_{1} g_{2}\right)^{2}} L_{c}^{2}
$$

From the eqn.(1), we have calculated TEM Modes, $\mathrm{W}_{00}, \mathrm{~W}_{01}, \mathrm{~W}_{10}, \mathrm{~W}_{11}$, and $\mathrm{W}_{20}$. TEM modes should be smaller than undulator gap to get laser out, thus these modes can be attainable for Undulator-1 in the IR-FEL System. In undulator-2, $\mathrm{W}_{00}, \mathrm{~W}_{01}, \mathrm{~W}_{10}$ exist within the undulator gap where beam waist is $0.01121 \mathrm{~m}$. These $\mathrm{TEM}_{\mathrm{pl}}$ Modes related with radial intensity of beam that is given by Laguerre polynomials. Radial intensity distributions are normalized to spot size of a Gaussian Beam profile. In our resonator system behavior shows Gaussian Mode shapes and partly covers Laguerre polynomials - as found above equations - $\mathrm{TEM}_{00}, \mathrm{TEM}_{01}, \mathrm{TEM}_{10}$, and $\mathrm{TEM}_{11}$ for undulator-1. In Figure 3, possible TEM Modes for undulator-1 are displayed while Figure 4 shows existing TEM Modes for undulator-2 in the IR-FEL facility.

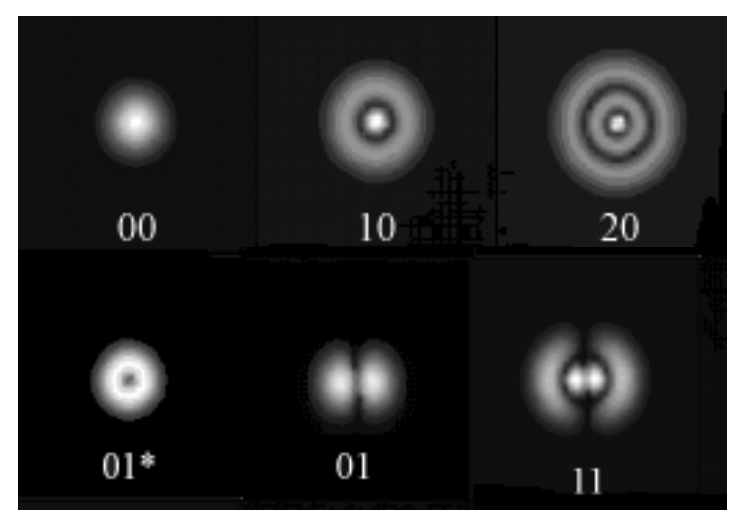

Figure 3: Possible TEM $00, \mathrm{TEM}_{01}, \mathrm{TEM}_{10}$, and TEM 11 Modes for undulator-1, U25

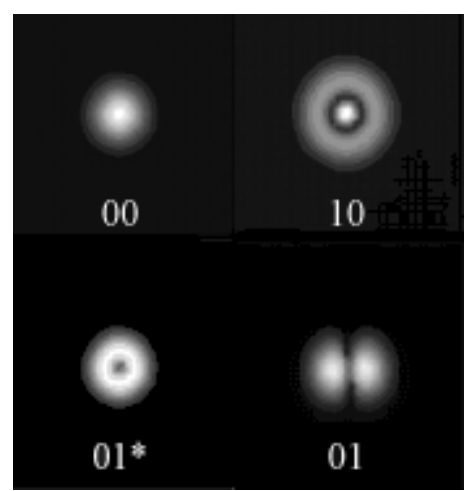

Figure 4: Possible TEM ${ }_{00}$, TEM $_{01}$, and TEM 10 Modes for undulator-1, U90 
Rayleigh length is obtained as $2.078 \mathrm{~m}$ for undulator- 2 with the stability parameter, $\mathrm{g}_{0}=$ -0.77 . By using $g_{0}$, beam spot size can be determined from the equation,

$$
\omega_{1}=\sqrt{\frac{L_{c} \lambda_{R}}{\pi}}\left(\frac{g_{2}}{g_{1}\left(1-g_{1} g_{2}\right)}\right)^{\frac{1}{4}}
$$

Minimum spot size for the lowest order TEM Mode occurs while Rayleigh length $\left(Z_{R} \approx L_{u} / 2\right.$ ) for $\mathrm{L}_{u}$. Other resonator and mirror parameters such as radius of curvature of the mirrors and spot size on mirrors are obtained by the equations,

$$
\begin{aligned}
& R(z)=z\left[1+\left(\frac{\pi w_{0}^{2}}{\lambda z}\right)^{2}\right] \\
& \omega^{2}(0)=\omega_{0}^{2}=\frac{\lambda_{R}}{2 \pi} \sqrt{L_{c}\left(2 R-L_{c}\right)}
\end{aligned}
$$

The results of the Genesis 1.3 Code, we able to obtained the power for only single pass with respect to Rayleigh length, shown in Figure 5 while the parameters are listed as a result of the Genesis 1.3 output file:

$\begin{array}{lccccc}\text { power } & \text { increment } & \text { p_mid } & \text { phi_mid } & \text { r_size } & \text { energy } \\ 9.9959 \mathrm{E}+00 & -1.6531 \mathrm{E}-02 & 2.9892 \mathrm{E}-02 & -6.7499 \mathrm{E}-02 & 6.8193 \mathrm{E}-04 & 3.9883 \mathrm{E}-10 \\ 9.8616 \mathrm{E}+00 & -5.4113 \mathrm{E}-01 & 2.1339 \mathrm{E}-02 & -1.3073 \mathrm{E}-01 & 6.8716 \mathrm{E}-04 & 4.4602 \mathrm{E}-10 \\ 9.5494 \mathrm{E}+00 & -1.2865 \mathrm{E}+00 & 1.1439 \mathrm{E}-02 & 1.1761 \mathrm{E}-01 & 6.9906 \mathrm{E}-04 & -8.6112 \mathrm{E}-10 \\ 9.2830 \mathrm{E}+00 & -1.1320 \mathrm{E}+00 & 1.9548 \mathrm{E}-02 & 6.7726 \mathrm{E}-01 & 7.0998 \mathrm{E}-04 & -3.1085 \mathrm{E}-09 \\ 9.4808 \mathrm{E}+00 & 8.4366 \mathrm{E}-01 & 5.8885 \mathrm{E}-02 & 6.5672 \mathrm{E}-01 & 7.0414 \mathrm{E}-04 & -3.6990 \mathrm{E}-09 \\ 1.0377 \mathrm{E}+01 & 3.6126 \mathrm{E}+00 & 1.1207 \mathrm{E}-01 & 3.3753 \mathrm{E}-01 & 6.7614 \mathrm{E}-04 & 4.3195 \mathrm{E}-10 \\ 1.1644 \mathrm{E}+01 & 4.6086 \mathrm{E}+00 & 1.3444 \mathrm{E}-01 & -8.7148 \mathrm{E}-02 & 6.4360 \mathrm{E}-04 & 9.5036 \mathrm{E}-09\end{array}$

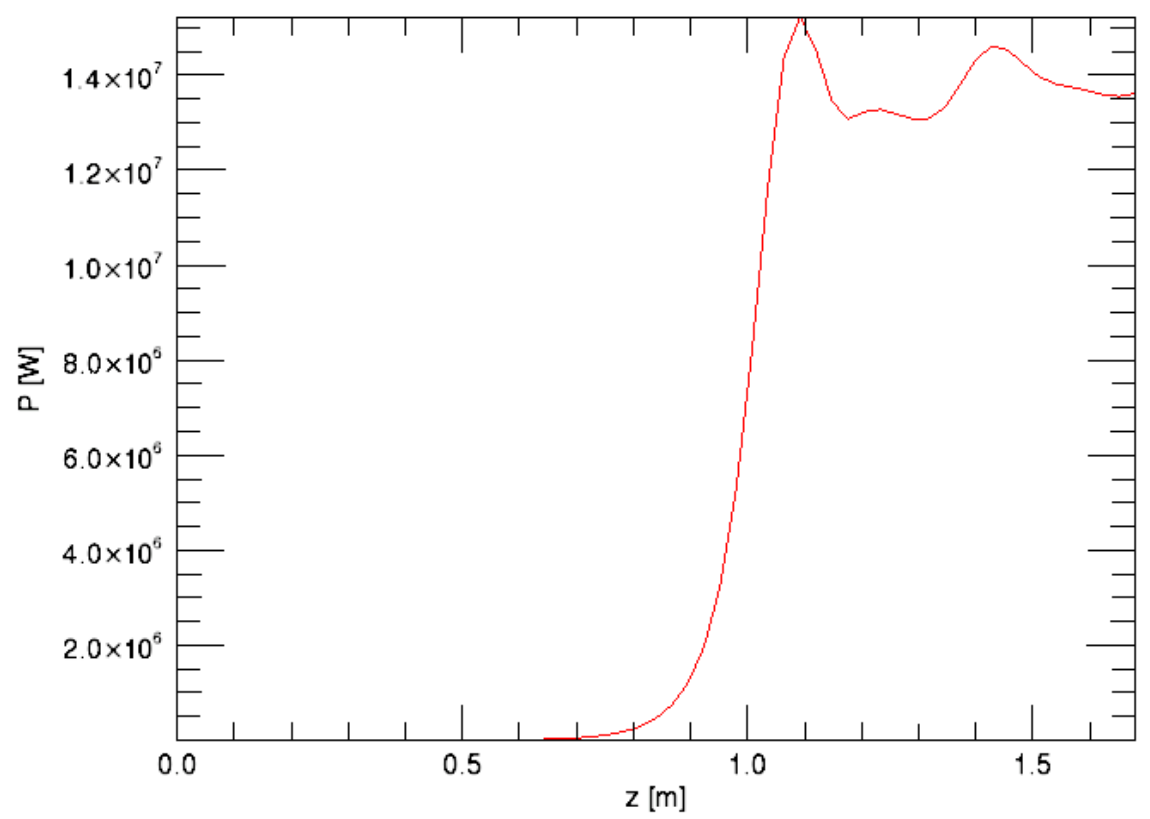

Figure 5: Power for single pass in the resonator for U25

To reduce loss of laser light between mirrors inside resonator and since IR-FEL facility 
covers very wide range of wavelength, waveguide system needs to be considered and placed inside the resonator. In the future studies, waveguide selection will be concerned. Main parameters of the optical resonator systems are calculated as in Table 2.

Stability condition for a stable resonator is given as:

$$
0<\left(1-\frac{L_{c}}{R_{1}}\right)\left(1-\frac{L_{c}}{R_{2}}\right)<1
$$

This condition is derived from a paraxial ray tracing in a periodic convergent lens sequence where $g_{1}=1-L_{c} / R_{1}$ and $g_{2}=1-L_{c} / R_{2}$. As one can see from the Figure 6-(a), multiplication of the stability parameters in our IR-FEL facility, $g_{1}$ and $g_{2}$, is housed in the stable area which is shaded. Stability criterion, $\mathrm{g}_{1} \times \mathrm{g}_{2}$, is found 0.731 .

Table 2: The main parameters of optical resonators.

\begin{tabular}{|l|c|c|}
\hline Parameter & Resonator-1 & Resonator-2 \\
\hline Undulator period $[\mathrm{mm}]$ & 25 & 90 \\
\hline Undulator length $[\mathrm{m}]$ & 1.50 & 3.6 \\
\hline Optic cavity length, $\mathrm{L}_{\mathrm{c}}[\mathrm{m}]$ & \multicolumn{2}{|c|}{11.53} \\
\hline Resonator Type & Symmetric, concentric \\
\hline $1^{\text {st }}$ Mirror, radius of curvature, $\mathrm{R}_{1}[\mathrm{~m}]$ & 5.92 & 6.51 \\
\hline $2^{\text {nd }}$ Mirror, radius of curvature, $\mathrm{R}_{2}[\mathrm{~m}]$ & 5.92 & 6.51 \\
\hline Stability criterion $\left(\mathrm{g}_{1}, \mathrm{~g}_{2}\right)$ & -0.9476 & -0.7711 \\
\hline Rayleigh length, $\mathrm{Z}_{\mathrm{R}}[\mathrm{m}]$ & 0.97 & 2.07 \\
\hline Mirror Material & $\mathrm{Au} / \mathrm{Cu}$ & $\mathrm{Au} / \mathrm{Cu}$ \\
\hline Radius of out coupling hole $[\mathrm{mm}]$ & $0.5 / 2$ & $0.5 / 2$ \\
\hline
\end{tabular}

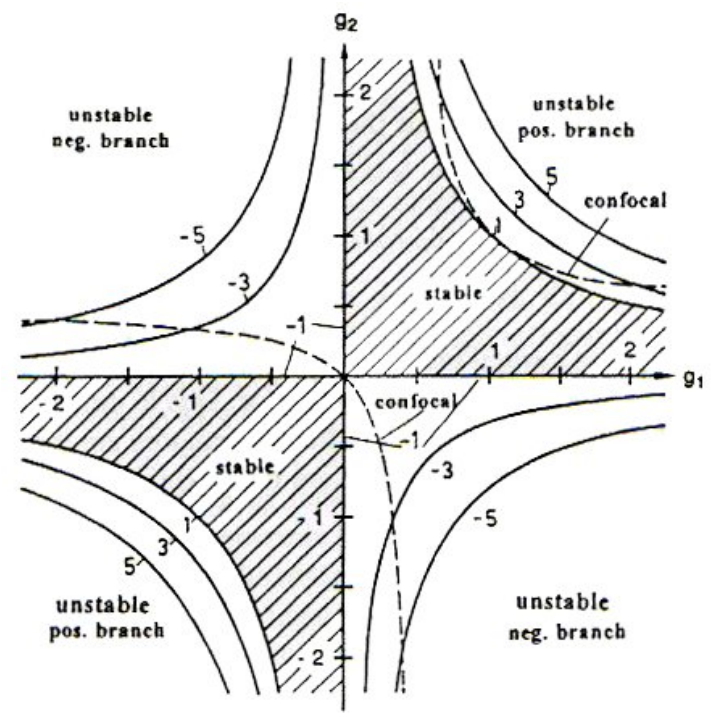

(a)

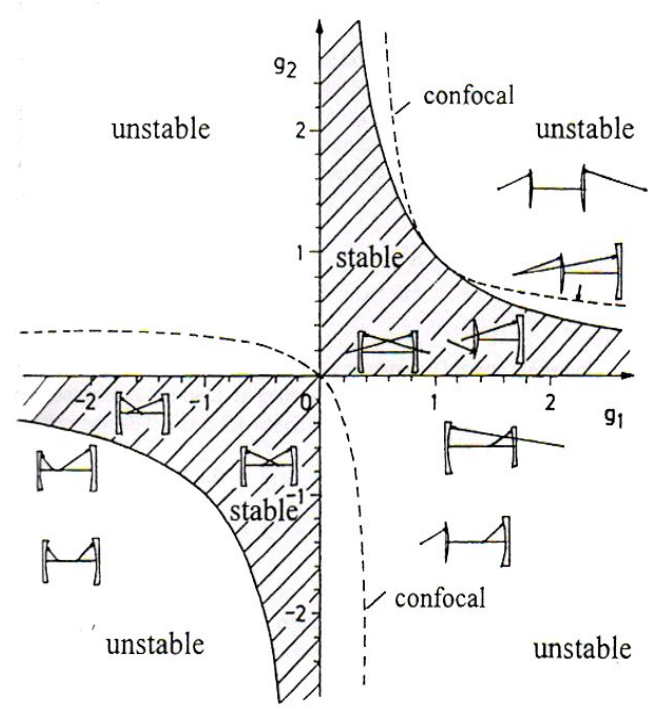

(b)

Figure 6: Stability diagrams, (a) shows detailed stability criteria for stable and unstable area while (b) shows resonator types corresponding stable and unstable regions 


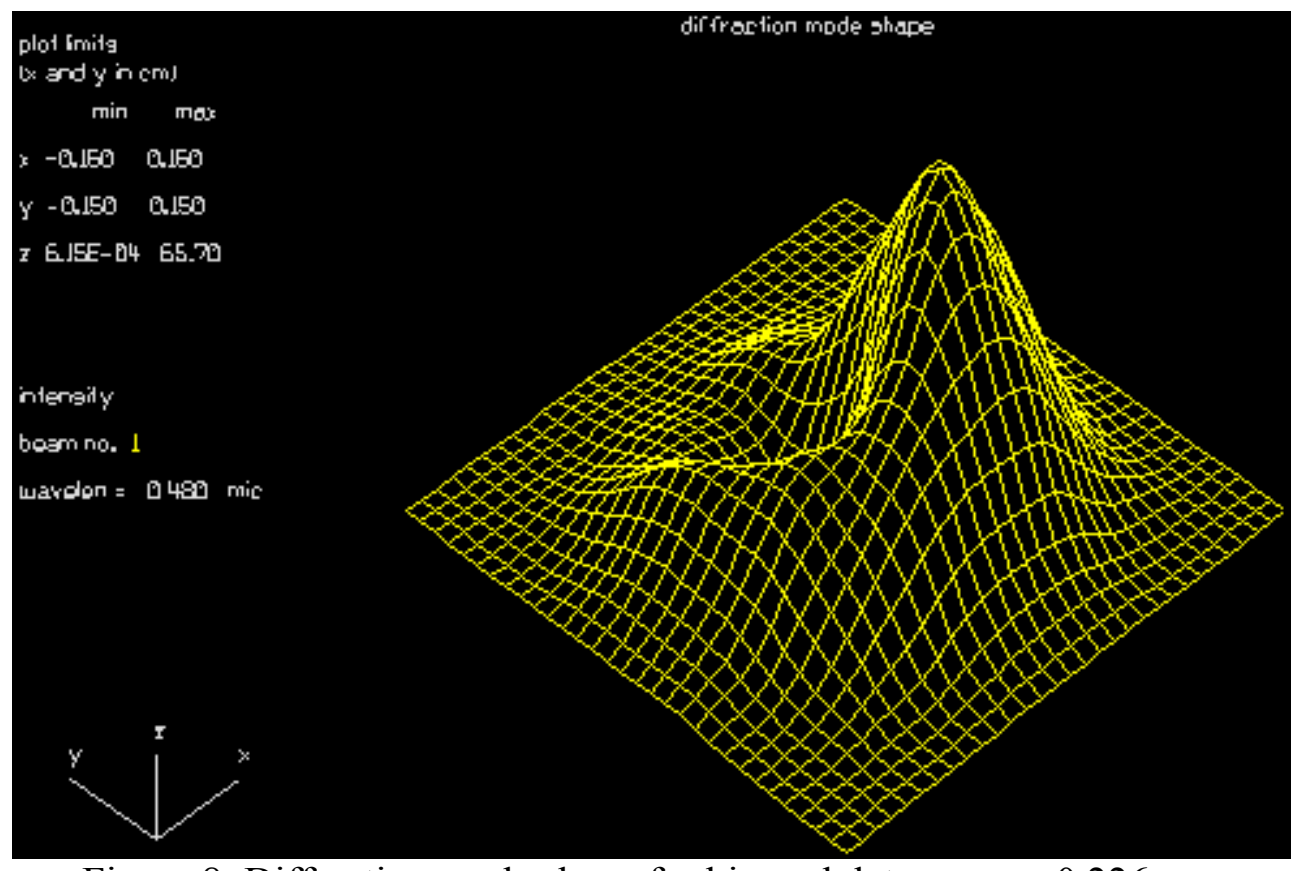

Figure 8: Diffraction mode shape for big undulator gap as $0.226 \mathrm{~cm}$.

Figure 7 and 8 show diffraction losses for $1.5 \mathrm{~m}$ undulator wavelength of silver mirror coating with .126 and $.226 \mathrm{~cm}$. undulator gap respectively. Shape becomes wider and covers high TEM Modes for bigger undulator gap.

\section{INFRARED-FREE ELECTRON LASER}

After calculation of the parameters, Lc, Lu, f, R, $Z_{R}, g_{1,2}, \omega$, TEM Modes, using intensity figures of these modes, small signal gain can be calculated by integrating the area under the curve either analytically or by programming. Using small signal gain, considering mirror hole $0.5-2 \mathrm{~mm}$, the following equations can be evaluated as:

$$
g_{0}=\frac{4 \pi}{\gamma} \frac{\hat{I}}{I_{0}} \frac{\lambda L_{u}}{\sum_{E}} f\left(\frac{\Delta \omega}{\omega}\right)_{0}^{-2} F(\xi)
$$

where $\quad f=\frac{\sum_{E}}{\sum_{L}}$ filling factor, $I_{0}=1.7 \times 10^{4} \mathrm{~A}$ Alfven current

homogen band width: $\left(\frac{\Delta \omega}{\omega}\right)=\frac{1}{2 N}$

where $\mathrm{N}$ stands for number of periods in the undulator and in the equation $8, \xi$ shows undulator parameter which is related to the Bessel Functions. Cylindrical Bessel functions $\mathrm{J}_{0,1}$ are plugged into define the undulator behavior. It differs for helical and linear undulator. We used planar undulator in our calculations since planar undulator is chosen for IR-FEL system. Following equation, the first part shows helical and second part related for linear undulator. In order to find small signal gain, second part is used 


$$
F(\xi)=\left\{\begin{array}{l}
\frac{1}{2} \frac{k^{2}}{1+k^{2}} \\
\frac{1}{2} \frac{k^{2}}{1+k^{2}}\left(J_{0}\left(\frac{k^{2}}{2\left(1+k^{2}\right)}\right)\right)-J_{1}\left(\left(\frac{k^{2}}{2\left(1+k^{2}\right)}\right)\right)^{2}
\end{array}\right.
$$

where

$$
\begin{aligned}
& J_{m}(x)=\sum_{n=0}^{\infty}(-1)^{n} \frac{1}{n !(m+n) !}\left(\frac{x}{2}\right)^{m+2 n} \\
& J_{0}(x)=\sum_{n=0}^{\infty}(-1)^{n} \frac{1}{n ! n !}\left(\frac{x}{2}\right)^{2 n}=1-\left(\frac{x}{2}\right)^{2}+\frac{1}{2 !^{2}}\left(\frac{x}{2}\right)^{4}+\ldots \ldots . .
\end{aligned}
$$

For the Concentric resonator, the Filling Factor is greater than (for $\lambda_{\mathrm{u}}=2.5 \mathrm{~cm}: \lambda=2.5-27$ $\mu \mathrm{m}$ and for $\left.\lambda_{\mathrm{u}}=9 \mathrm{~cm}: \lambda=11-250 \mu \mathrm{m}\right) 50 \%$ in the output FEL wavelength. To obtain FEL in 2.5-250 microns range using $15-40 \mathrm{MeV}$ energy electron beam inclined us to use undulators that have 2.5 and $9 \mathrm{~cm}$ period lengths which we call undulator- 1 and 2 respectively. In calculations, we have taken the electron beam to have 1,6 $\mathrm{mA}$ average current, $1 \mathrm{ps}$ bunch length, $13 \mathrm{MHz}$ repetition rate, $15 \mathrm{~mm} . \mathrm{mrad}$ normalized transverse emittance and $100 \mathrm{keV}$.ps normalized longitudinal emittance. Using small signal gain $\left(\mathrm{g}_{0}\right)$, total gain can be calculated with the following relation,

$$
G_{\max }=0.85 * g_{0}+0.19 * g_{0}^{2}+4.12 * 10^{-3} * g_{0}^{3}
$$

For IR-FEL system, one can obtain small single gain as 0.006175 and gain for many pass is approximately found as 0.00645 for undulator- 1 with $2.5 \mathrm{~cm}$ wavelength. To find power after many pass studies with Modified Genesis Code still continues. Electron beam energy spread, finite emittance, longitudinal slippage and Chesworth filling method correction factors are utilized for calculating the ideal gain. It is assumed $\% 10$ cavity losses, $120 \mathrm{pC}$ bunch charges, \%0.1 beam energy spread and $15 \mathrm{~mm} . \mathrm{mrad}$ RMS transverse emittance for both undulator simulations. Bunch lengths and cavity detuning were assumed to be different for better coupling in the resonators.

Table 3:Expected Main Parameters of IR-FEL Facility for 1,6 mA average beam current

\begin{tabular}{|l|c|c|}
\hline Parameter & Undulator-1 & Undulator-2 \\
\hline Wavelength $[\mu \mathrm{m}]$ & $2.5-27$ & $11-250$ \\
\hline Micropulse repetition rate $[\mathrm{MHz}]$ & 13 & 13 \\
\hline Max. Peak Power $[\mathrm{MW}]$ & 5 & 2.5 \\
\hline Average Power $[\mathrm{W}]$ & $0.1-40$ & $0.1-30$ \\
\hline Max. Pulse energy $[\mu \mathrm{J}]$ & 10 & 8 \\
\hline Pulse length $[\mathrm{ps}]$ & $1-10$ & $1-10$ \\
\hline
\end{tabular}


The output IR-FEL wavelength is given by an analytical well known expression:

$$
\lambda_{r}=\frac{\lambda_{u}}{2 \gamma^{2}}\left(1+a_{u}^{2}\right)
$$

where $\lambda_{u}$ is undulator period length, $a_{u}$ is rms undulator strength parameter, and $\gamma$ is Lorentz factor that is a measure of electron energy. Calculated Main parameters of FEL for undulator-1 and 2 with $120 \mathrm{pC}$ bunch charge are given in Table 3. Calculation is based on analytics in addition to OPC and GENESIS simulation codes.

\section{RESULTS AND CONCLUSION}

In the IR-FEL System, resonator parameters are calculated with both analytically and Genesis 1.3, OPC Codes. Optimized results are obtained and these results will follow the design tests in the IR-FEL system soon. Mirror alignments and waveguide placement in resonator become important since the laser wavelength range is very wide. Waveguide is based on Gaussian Modes as given in this study. Mirror cooling, surface roughness will also be tested before assembly done with He-Ne visible laser.

Construction Free electron laser system is being done for the first time in Turkey. The IR-FEL System will be the first step of the accelerator complex in Turkey - TAC (Turkish Accelerator Complex). Other parts of the complex cover synchrotron radiation, particle factory, proton accelerator and SASE-FEL. After commissioning is completed for the first step at 2013, many applications that are using IR-FEL become more effective and popular than ever before. Some of these applications that are interested, Fourier Transform of IR studies, industrial chemical catalyzes, material science researches, surface characterization studies, military, medical applications, dentistry and in each part of the medicine industry.

\section{Acknowledgement}

This work is supported by Turkish Atomic Energy Authority (TAEA), Turkish State Planning Organization under grant no. DPT2006K-120470, and TÜBİTAK under grant no. 105T442. I thank to İ. Y1ldız and N. Kenar for valuable discussions.

\section{References}

1. F. Ciocci, G. Dattoli, A. Torre, A. Renieri, Insertion Devices for Synchrotron Radiation and Free Electron Laser (World Scientific, 2000), ISBN 981-02-3832-0.

2. Tyte D. C., In Advances in Quantum Electronics', Vol.1, (D.W. Goodwin, ed., Academic Press, New York, 1970).

3. Rigrod W. W., Journal of Applied Physics, V34, pp. 248 (1965).

4. Kogelnik H., Li, T., Proceedings of the IEEE, pp. 97 (1966).

5. N. Hodson, Laser Resonators and Beam Properties, (Optical Sciences, 2004).

6. GLAD Code, http://www.aor.com/anonymous/pub/theory.pdf

7. Genesis 1.3 Code, http://corona.physics.ucla.edu/ reiche/

8. Optical Propagation Code, http://lpno.tnw.utwente.nl/project.php?projectid=21\&submenu=16 